\title{
Phase II Study of Dovitinib in Patients with Castration-Resistant Prostate Cancer (KCSG-GU11-05)
}

\author{
Yoon Ji Choi, MD, PhD' \\ Hye Sook Kim, MD, PhD' \\ Se Hoon Park, MD, PhD² \\ Bong-Seog Kim, MD, PhD 3 \\ Kyoung Ha Kim, MD ${ }^{4}$ \\ Hyo Jin Lee, MD, PhD ${ }^{5}$ \\ Hong Suk Song, MD, PhD ${ }^{6}$ \\ Dong-Yeop Shin, MD, PhD7,8 \\ Ha Young Lee, MD, $\mathrm{PhD}{ }^{9}$ \\ Hoon-Gu Kim, MD, PhD ${ }^{10}$ \\ Kyung Hee Lee, MD, PhD ${ }^{11}$ \\ Jae Lyun Lee, MD, PhD² \\ Kyong Hwa Park, MD, PhD ${ }^{1}$
}

*A list author's affiliations appears at the end of the paper.

\begin{abstract}
Purpose
Fibroblast growth factor (FGF) signals are important in carcinogenesis and progression of prostate cancer. Dovitinib is an oral, pan-class inhibitor of vascular endothelial growth factor receptor (VEGFR), platelet-derived growth factor receptor, and fibroblast growth factor receptor (FGFR). We evaluated the efficacy and toxicity of dovitinib in men with metastatic castration resistant prostate cancer (mCRPC).
\end{abstract}

\section{Materials and Methods}

This study was a single-arm, phase II, open-label, multicenter trial of dovitinib $500 \mathrm{mg} /$ day (5-days-on/2-days-off schedule). The primary endpoint was 16-week progression-free survival (PFS). Secondary endpoints were overall survival (OS), toxicity and prostate-specific antigen (PSA) response rate. Biomarker analyses for VEGFR2, FGF23, and FGFR2 using multiplex enzyme-linked immunosorbent assay was performed.

\section{Results}

Forty-four men were accrued from 11 hospitals. Eighty percent were post-docetaxel. Median PSA was $100 \mathrm{ng} / \mathrm{dL}$, median age was 69, 82\% had bone metastases, and 23\% had liver metastases. Median cycles of dovitinib was 2 (range, 0 to 33). Median PFS was 3.67 months (95\% confidence interval [Cl], 1.36 to 5.98) and median OS was 13.70 months ( $95 \% \mathrm{Cl}, 0$ to 27.41 ). Chemotherapy-naïve patients had longer PFS (17.90 months; $95 \% \mathrm{Cl}, 9.23$ to 28.57) compared with docetaxel-treated patients ( 2.07 months; $95 \% \mathrm{Cl}, 1.73$ to 2.41; $\mathrm{p}=0.001)$ and the patients with high serum VEGFR2 level over median level $(7,800 \mathrm{pg} / \mathrm{mL})$ showed longer PFS compared with others (6.03 months [95\% Cl, 4.26 to 7.80] vs. 1.97 months [95\% $\mathrm{Cl}, 1.79$ to 2.15], $\mathrm{p}=0.023$ ). Grade 3 related adverse events were seen in $40.9 \%$ of patients. Grade 1-2 nausea, diarrhea, fatigue, anorexia, and all grade thrombocytopenia are common.

\section{Conclusion}

Dovitinib showed modest antitumor activity with manageable toxicities in men with mCRPC. Especially, patients who were chemo-naïve benefitted from dovitinib.

\section{Key words}

Dovitinib, Castration-resistant prostatic neoplasm, Biomarkers
Correspondence: Kyong Hwa Park, MD, PhD Division of Oncology/Hematology,

Department of Internal Medicine,

Korea University Anam Hospital, 73 Inchon-ro, Seongbuk-gu, Seoul 02841, Korea

Tel: 82-2-920-6841

Fax: 82-2-926-4534

E-mail: khpark@korea.ac.kr 


\section{Introduction}

Prostate cancer is the most common cancer among men worldwide [1]. In Korea, prostate cancer has been continually increasing in prevalence and is now the fourth most commonly diagnosed cancer and the eighth leading cause of cancer-related death in men [2].

Growth factor signals are important in carcinogenesis and progressiton of prostate cancer, and fibroblast growth factors (FGF) have important roles in this regard. FGF ligands (FGF1, -2, -6, -8, and -17) and FGF receptors (FGFR1 and FGFR4) are significantly overexpressed in prostate cancer [3-6]. Recent studies have demonstrated that critical roles of the FGF family members are mediated by the signaling between epithelial and stromal compartments, which promotes the epithelial-mesenchymal transition $[7,8]$. Moreover, FGF-2 is a mediator of second-wave angiogenesis and tumor progression in men during the formation of castration-resistant tumors [9]. Therefore, inhibition of signaling via the FGF axis might be a viable strategy for the treatment of castration-resistant prostate cancer.

Dovitinib, an oral multitargeted receptor tyrosine kinase (RTK) inhibitor, potently inhibits class III, IV, and V RTKs, showing biochemical $\mathrm{IC}_{50}$ values $<20 \mathrm{nmol} / \mathrm{L}$ for RTKs that include vascular endothelial growth factor receptors (VEGFR-1, VEGFR-2, and VEGFR-3), platelet-derived growth factor receptor- $\beta$, fibroblast growth factor receptors (FGFR1, FGFR-2, and FGFR-3), fetal liver tyrosine kinase receptor 3, KIT Ret, TrkA, and csf-1. Due to its unique inhibitory activity on FGF pathways, dovitinib has significant activity in a variety of tumor xenograft models in athymic mice, including acute myeloid leukemia, multiple myeloma, and colon- and prostate-derived models [10].

Castration-resistant prostate cancers (CRPCs) are one of the challenges in oncology practice. Although there have been advances in chemotherapy [11], hormonal agents [12], and immunotherapeutics [13], CRPC patients still have limited life expectancy. There is an urgent need to identify therapeutic targets and clinical development of target agents for the treatment of CRPC. To this end, sorafenib has been tested in phase II studies [14]. However, the clinical efficacy was very limited. The low efficacy of sorafenib might be partly explained by the lower potency in inhibition of RTKs. Considering nanomolar concentration range of $\mathrm{IC}_{50}$ for dovitinib compared with micrololar concentration for other multityrosine kinase inhibitors (TKIs) [15], the efficacy of dovitinib should be evaluated in CRPC patients.

The present phase 2 trial evaluated whether dovitinib would improve progression-free survival (PFS) in men with progressive CRPC.

\section{Materials and Methods}

\section{Study design and population}

This multi-center, single-arm, open-label, phase II study evaluated the efficacy and toxicity of dovitinib in CRPC.

Patients were eligible if they were $\geq 20$ years of age, had a histological or cytological diagnosis of prostate cancer with documented metastases, and prostate-specific antigen (PSA) and/or radiographic progression despite receiving luteinizing hormone releasing hormone analogue therapy or undergoing orchiectomy, and serum testosterone level $\leq 50 \mathrm{ng} / \mathrm{dL}$. Patients with only non-measurable lesions could be enrolled if evaluable lesions were present. Patients had to be exposed to two lines or less of previous cytotoxic chemotherapy, have an Eastern Cooperative Oncology Group performance of $0-2$, and have adequate bone marrow, renal, and hepatic function indicated by a neutrophil count $\geq 1.5 \times 10^{9} / \mathrm{L}$, platelet count $\geq 75 \times 10^{9} / \mathrm{L}$, hemoglobin $>8 \mathrm{~g} / \mathrm{dL}$, total bilirubin $\leq 1.5$ times the normal limit, alanine aminotransferase and aspartate aminotransferase $\leq 2$ times the upper limit of normal, and serum creatinine $\leq 1.5$ times the upper limit of normal (if values were borderline, the creatinine clearance had to be $\geq 30 \mathrm{~mL} / \mathrm{min}$ by Cockcroft and Gault formula).

Exclusion criteria included history of central nervous system metastasis, second primary malignancy within 3 years except for completely cured non-melanoma skin cancer, other systemic treatment (chemotherapy, immunotherapy, TKI, or monoclonal antibody) within 4 weeks, and any unstable medical condition.

\section{Treatment}

Dovitinib was administered at $500 \mathrm{mg}$ orally once daily for 5 consecutive days, followed by a 2-day rest, with each cycle consisting of 28 days. Treatment was discontinued if patients had disease progression, intolerable toxicities, or withdrew. Doses were modified based on the worst grade of toxicity according to the protocol, but patients who had two dose reductions (300 mg per day) and who experienced toxicity requiring a third dose reduction were discontinued from the study treatment. Patients who interrupted treatment for more than 21 days were discontinued.

\section{Evaluation of response and adverse events}

Tumor response was assessed by computed tomography or magnetic resonance imaging and bone scan with Response Evaluation Criteria in Solid Tumor (RECIST) ver. 1.1 every 8 weeks during treatment. Progression of osseous disease was indicated by bone scans showing two or more new lesions, 
with the requirement of a confirmatory scan performed 6 or more weeks later showing additional new lesions according to Prostate Cancer Working Group 2 (PCWG2). Toxic effects were evaluated at each visit using Common Terminology Criteria for Adverse Events ver. 4.0. The primary endpoint of the study was the 16-week PFS rate, defined as the proportion of patients showing complete response (CR), partial response (PR), or stable disease (SD) in week 16 after treatment started. Secondary endpoints were PFS, objective response rate, PSA response rate, overall survival (OS), and toxicity.

\section{Biomarker analysis}

We planned to collect blood samples before and after two cycles of study treatment for biomarker study. The blood samples were kept at room temperature within 2 hours to allow clotting and then centrifuged at 3,000 $\times g$ for 5 minutes. The serum was collected at stored at $-80^{\circ} \mathrm{C}$ until analysis. Serum levels of VEGFR2 and FGF23 were measured using MILLIPLEX MAP kits (Millipore Corporation, Billerica, MA). Serum levels of FGFR2 were measured using an ELISA kit (Biorbyt Co., Cambridge, UK). Serum levels were compared with tumor response as an exploratory endpoint.

\section{Statistical analyses}

The study was designed to rule out a $30 \%$ probability of 16-week PFS while targeting a 50\% probability of patients having 16-week PFS at the 0.10 significance level with a power of 0.90 . The study required a total of 39 evaluable patients to demonstrate this hypothesis. With a $10 \%$ dropout rate, 44 patients were enrolled to ensure 39 evaluable patients. The probability of survival was estimated by the Kaplan-Meier method, and the log-rank test with 95\% confidence interval (CI) was used to compare survival curves. A p-value $<0.05$ was considered statistically significant. Analyses were performed using SPSS ver. 24.0 (IBM Corp., Armonk, NY).

\section{Ethical statement}

The protocol was approved by the institutional review boards of participating institutions and Korea Cancer Study Group (KCSG-GU11-05) and registered at ClinicalTrials.gov (NCT01741116). Written informed consents were obtained.
Table 1. Baseline characteristics

\begin{tabular}{|cc|}
\hline Characteristic & No. $(\%)(\mathbf{n}=44)$ \\
\hline Age, median (range, $\mathbf{y r})$ & $69(57-88)$ \\
\hline ECOG performance status & \\
\hline 0 & $4(9.3)$ \\
1 & $34(79.1)$ \\
2 & $5(11.6)$ \\
Gleason score & \\
$\leq 6$ & $1(2.3)$ \\
\hline 7 & $4(9.1)$ \\
\hline$\geq 8$ & $36(81.8)$ \\
\hline Unknown & $3(6.8)$ \\
\hline Stage at diagnosis & $15(34.1)$ \\
\hline Localized & $27(61.4)$ \\
\hline Metastatic & $2(4.5)$ \\
\hline Unknown & $3.66(2.75-4.57)$ \\
\hline Time to CRPC, average (95\% CI, yr) & \\
Previous treatment & $16(36.4)$ \\
\hline Surgery & $17(38.6)$ \\
\hline Radiation & $35(79.5)$ \\
\hline Chemotherapy & \\
\hline Metastatic sites & $36(81.8)$ \\
\hline Bone & $10(22.7)$ \\
\hline Regional lymph node & $25(56.8)$ \\
\hline Metastatic lymph node & $10(22.7)$ \\
\hline Liver & $100(0.02-1,247.55)$ \\
\hline PSA, median (range, ng/dL) & \\
\hline
\end{tabular}

ECOG, Eastern Cooperative Oncology Group; CRPC, castration resistant prostate cancer; $\mathrm{CI}$, confidence interval; PSA, prostate specific antigen.

Table 2. Response to dovitinib

\begin{tabular}{ll} 
Endpoint & Rate $\mathbf{( 9 5 \%}$ CI, \%) \\
\hline 16-Week PFS rate & $42.1(27.9-57.8)$ \\
Objective response rate & $12.5(5.0-28.1)$ \\
Disease control rate & $59.4(42.4-76.4)$ \\
PSA response rate & $12.9(1.3-25.4)$ \\
\hline
\end{tabular}

CI, confidence interval; PFS, progression-free survival; PSA, prostate specific antigen.

\section{Results}

\section{Patient characteristics}

Between 2012 and 2015, a total of 44 patients were enrolled from 11 hospitals in South Korea. Median age was 69 years 


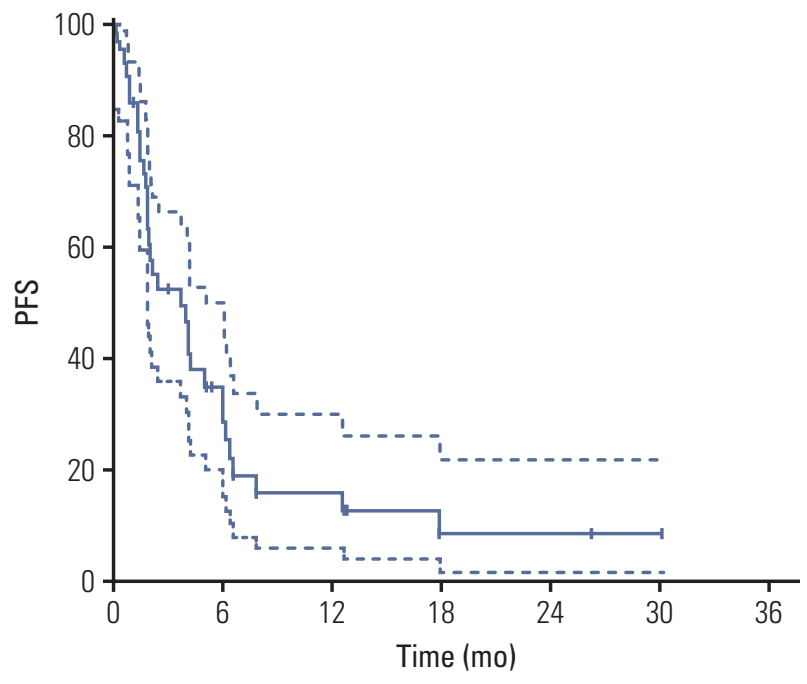

Fig. 1. Progression-free survival (PFS) (A) and overall survival (OS) (B). Dashed lines are the upper bound and lower bound of $95 \%$ confidence interval of Kaplan-Meier estimates.

(range, 57 to 88 years) and about $80 \%$ of the patients had already received chemotherapy. Docetaxel was included in all chemotherapy regimens. Eighty-two percent of patients had bone metastases and $23 \%$ had liver metastases. Their median PSA level was $100.00 \mathrm{ng} / \mathrm{dL}$ (range, 0.02 to 1,249.55 $\mathrm{ng} / \mathrm{dL}$ ) and time to CRPC was 3.66 years (95\% CI, 2.75 to 4.57). Baseline characteristics are shown in Table 1.

\section{Efficacy}

The response to dovitinib is described in Table 2. Sixteen of the 38 evaluable patients did not experience disease progression until week 16 of dovitinib treatment. The proportion of patients alive and progression free at 16-weeks (16-week PFS) was $42.1 \%$ (95\% CI, 27.9 to 57.8). There was a significant difference in 16-weeks PFS between chemotherapy-naïve patients $(87.5 \%$; 95\% CI, 57.1 to 100.0$)$ and post-docetaxel patients $(30.0 \%$; 95\% CI, 13.8 to $46.1 ; \mathrm{p}=0.03)$. A total of 32 patients were able to evaluate the tumor response with RECIST ver. 1.1. because 12 patients had unknown response data mostly due to withdrawal. One patient died from traffic accident. Of the patients who evaluated the response, no patients experienced CR, four patients had PR (12.5\%), 15 patients had SD (46.9\%), 13 patients $(40.6 \%)$ had progressive disease, and The objective response rate was $12.5 \%$ (95\% CI, 5.0 to 28.1). Follow-up PSA data was available for 31 patients. PSA response ( $\geq 50 \%$ decline) was observed in four patients, representing a PSA response rate of $12.9 \%$ (95\% CI, 1.3 to 25.4). The PSA decline was observed in $32.3 \%$ of patients.

With a median follow-up duration of 19.5 months by reverse Kaplan-Meier method, the median PFS was 3.67

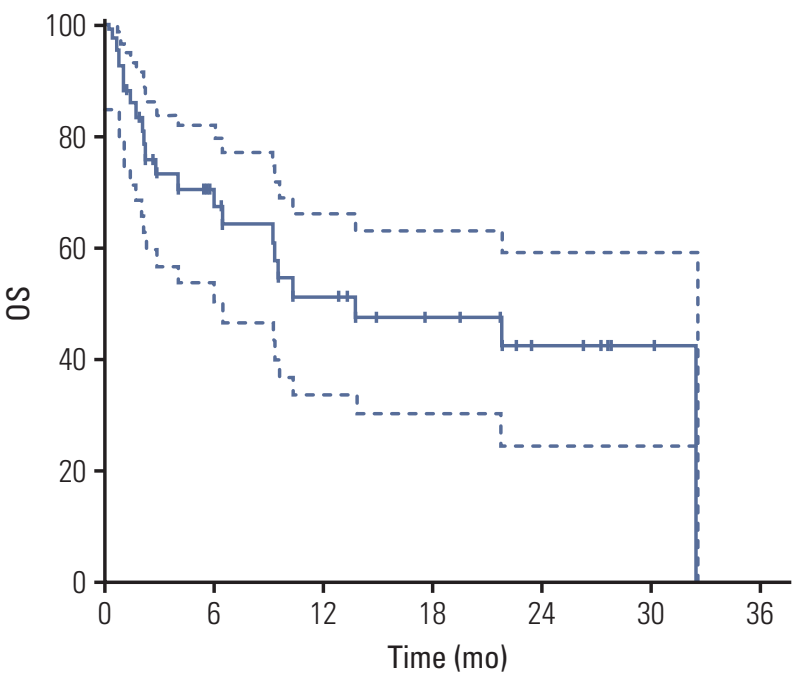

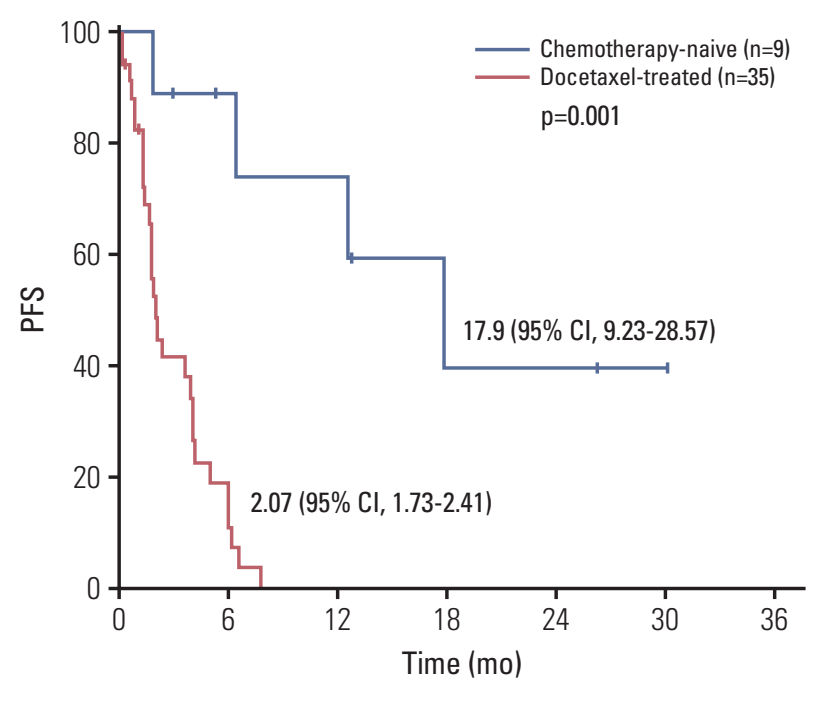

Fig. 2. Progression-free survival (PFS) according to history of chemotherapy. CI, confidence interval.

months (95\% CI, 1.36 to 5.98) and median OS was 13.70 months (95\% CI, 0 to 27.41) (Fig. 1). Chemotherapy-naïve patients had longer PFS (17.90 months; 95\% CI, 9.23 to 28.57) compared with docetaxel-treated patients (2.07 months; $95 \%$ CI, 1.73 to 2.41; $\mathrm{p}=0.001$ ) (Fig. 2). The duration of clinical benefit among 19 patients who experienced clinical benefit (CR, PR, and SD > 6 weeks) was much longer in chemotherapynaïve patients (Fig. 3). Some continued to benefit even after discontinuing treatment. 


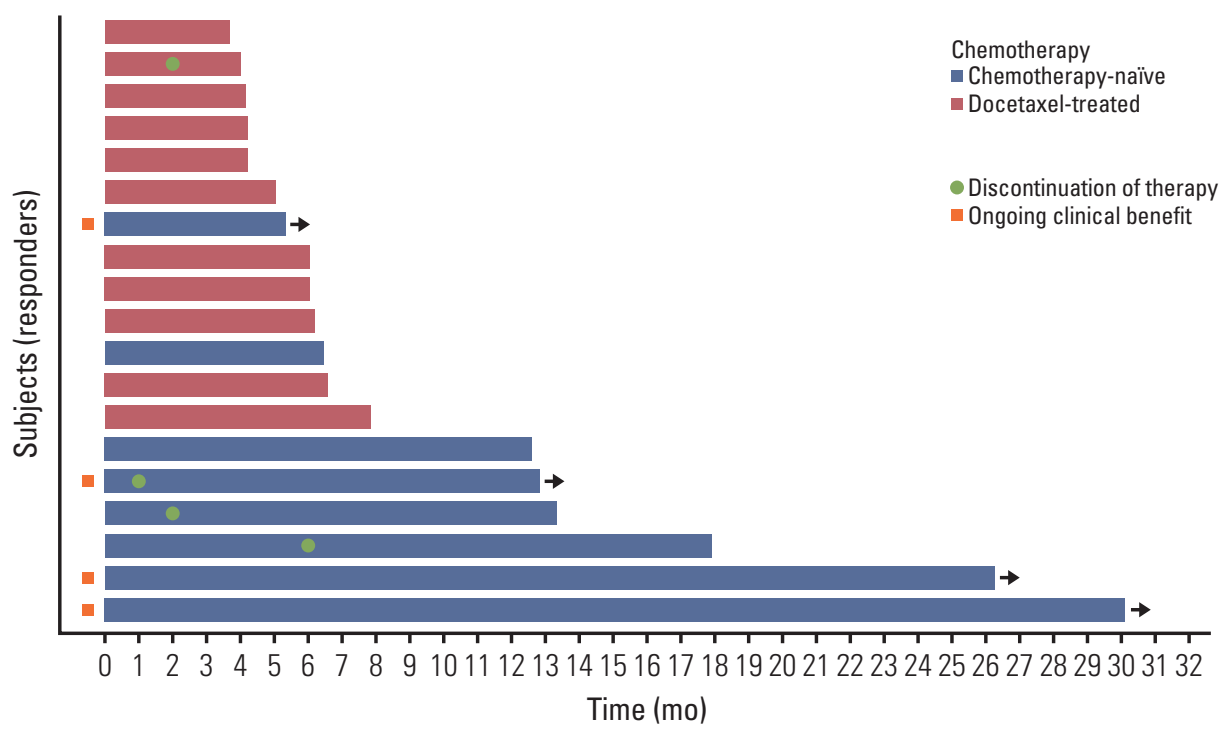

Fig. 3. Duration of clinical benefit.

Table 3. Summary of maximum common toxicity criteria

\begin{tabular}{lcc}
\hline Toxicity & All grade $(\mathbf{n}=\mathbf{4 2})$ & Grade $\mathbf{3 / 4}(\mathbf{n}=\mathbf{4 2})$ \\
\hline Laboratory & & \\
\hline Neutropenia & $21(50.0)$ & $3(7.1)$ \\
\hline Anemia & $26(61.9)$ & $2(4.8)$ \\
\hline Thrombocytopenia & $13(31.0)$ & $6(14.3)$ \\
\hline Bilirubin elevation & $3(7.1)$ & $2(4.8)$ \\
\hline Non-laboratory & & \\
\hline Nausea & $14(33.3)$ & $2(4.8)$ \\
\hline Anorexia & $16(38.1)$ & $2(4.8)$ \\
\hline Vomiting & $5(11.9)$ & 0 \\
\hline Diarrhea & $18(42.9)$ & $3(7.1)$ \\
\hline Fatigue & $9(21.5)$ & $2(4.8)$ \\
\hline
\end{tabular}

Values are presented as number $(\%)$.

\section{Treatment and toxicity}

The 44 enrolled patients received a total of 180 cycles of dovitinib. Median number of cycles administered was two (range, 0 to 33). About one-third of the cycles (57 of 180) were administered at reduced doses. Forty-two patients were evaluable for toxicity (Table 3). There was no death attributed to protocol treatment. Grade $3 / 4$ adverse events (AEs) were seen in $40.9 \%$ of patients with $7.0 \%$ of patients halting dovitinib treatment due to toxicity or withdrawal of consent. The most common related non-laboratory AEs included grade $1-2$ diarrhea $(42.9 \%)$, anorexia (38.1\%), nausea (33.3\%), and fatigue (21.5\%). Grade 3/4 thrombocytopenia (14.3\%) was the most common hematologic toxicity.

\section{Biomarker study}

Mean baseline plasma FGFR2 level was $56.0 \mathrm{pg} / \mathrm{mL}$ (range, 0 to $603.1 \mathrm{pg} / \mathrm{mL}$ ) and mean baseline plasma VEGFR2 level was $8,170.7 \mathrm{pg} / \mathrm{mL}$ (range, 2,787.4 to $15,497.4$ $\mathrm{pg} / \mathrm{mL}$ ) among the 34 patients whose baseline samples were available. We could not analyze FGF-23, because FGF-23 was not detected in most samples collected. Among the 18 patients whose follow-up samples were available, there was no significant changes from baseline including FGFR2 (48.7 $\mathrm{pg} / \mathrm{mL}$ vs. $76.9 \mathrm{pg} / \mathrm{mL}, \mathrm{p}=0.401)$ and VEGFR2 concentrations $(8,195.7 \mathrm{pg} / \mathrm{mL}$ vs. $8,942.1 \mathrm{pg} / \mathrm{mL}, \mathrm{p}=0.446)$ after treatment with dovitinib (Fig. 4). The patients with higher baseline VEGFR2 (more than median) showed longer PFS compared with others $(6.03 ; 95 \% \mathrm{CI}, 4.26$ to 7.80 vs. $1.97 ; 95 \% \mathrm{CI}$, 1.79 to 2.15 months, $p=0.023$ ) (Fig. 5). However, there were no differences of PFS according to baseline FGFR2 level and changes of FGFR2 and VEGFR2 levels after treatment.

\section{Discussion}

This phase II study was designed to evaluate the effect of dovitinib in the treatment of CRPC. The findings demonstrate that dovitinib has modest activity and a mostly acceptable safety profile in patients with CRPC.

Dovitinib showed a small but obvious effect. This might 

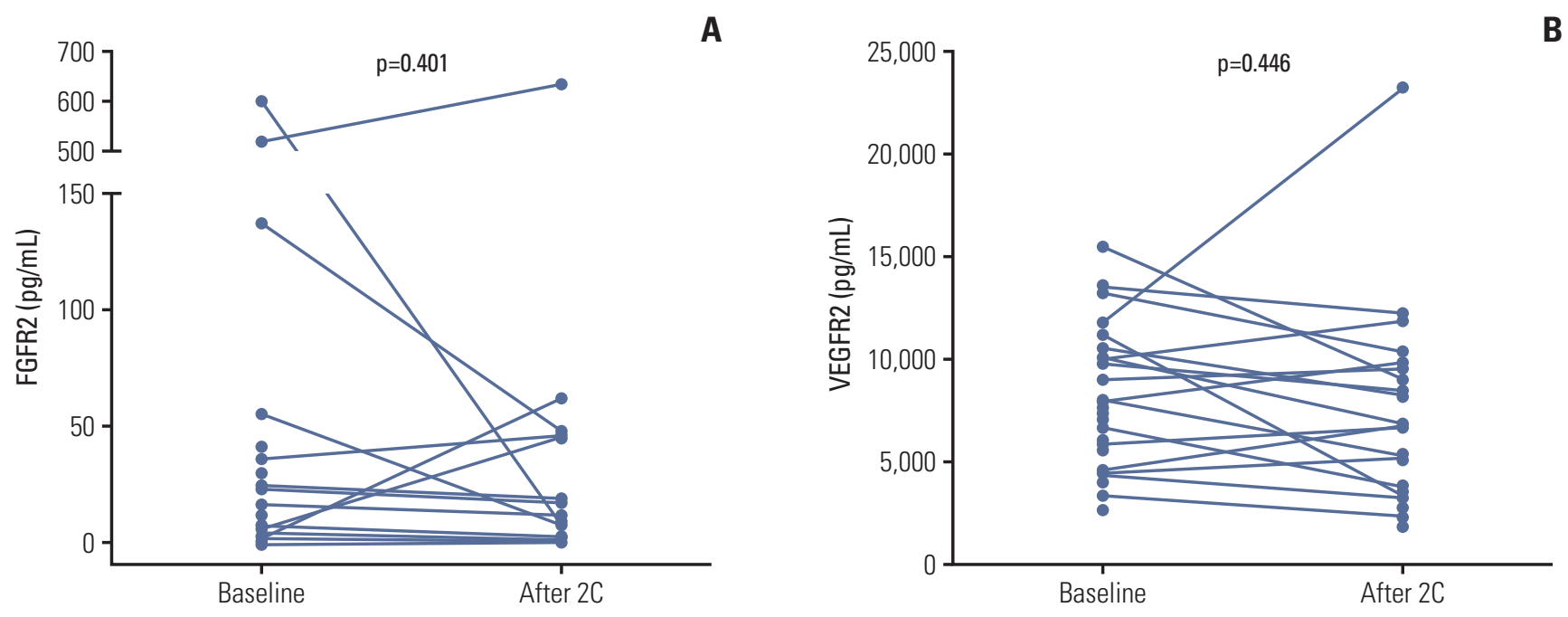

Fig. 4. Biomarker levels at baseline and after two cycles of dovitinib. (A) Fibroblast growth factor receptor 2 (FGFR2) levels. (B) Vascular endothelial growth factor receptor 2 (VEGFR2) levels.

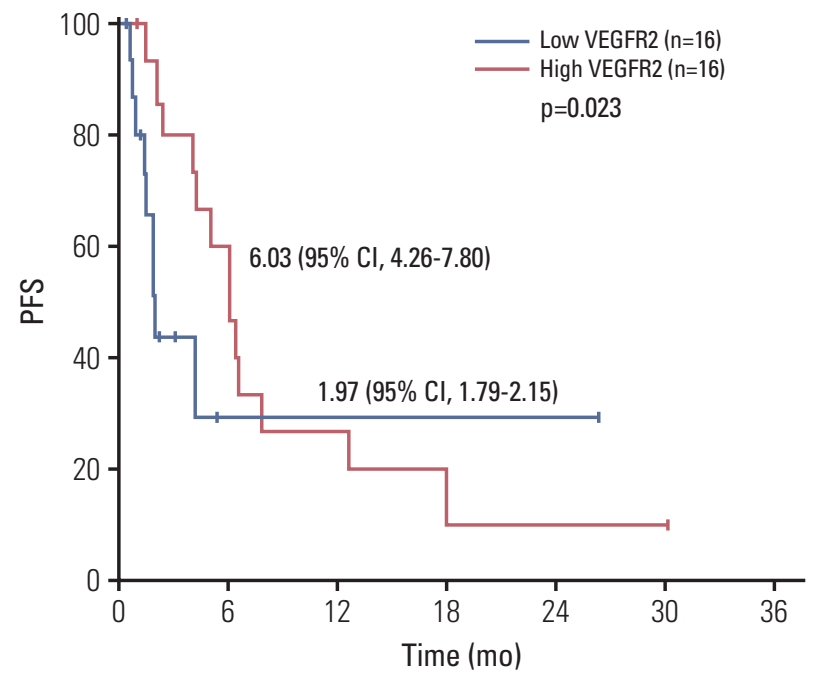

Fig. 5. Progression-free survival (PFS) according to baseline vascular endothelial growth factor receptor 2 (VEGFR2) levels. CI, confidence interval.

reflect the critical role of the FGF pathway in the development and progression of CRPC, as previous preclinical studies reported [7-9]. Another study also reported that upregulated FGFR1 expression was associated with transition of hormone-naïve prostate cancer to CRPC [16]. Presently, the 16-week PFS rate was $42.1 \%$ and the median PFS was 3.7 months (95\% CI, 1.36 to 5.98) in evaluable subjects. Despite the overall modest activity, chemotherapy-naïve patients, who accounted for $20 \%$ of all subjects, showed relatively good results. There is no evidence to date to explain the markedly better PFS in chemotherapy-naïve patients. Suggestions include the different natural course depending on the time elapsed since diagnosis of CRPC and variation by mutation after undergoing docetaxel chemotherapy.

Most patients tolerated the dovitinib treatment. However, some patients experienced severe side effects that necessitated the end of treatment. Grade $3 / 4$ thrombocytopenia was relatively frequent $(14.8 \%)$, perhaps because most patients enrolled had bone metastases and were elderly. Dose reductions should be considered for CRPC patients with disseminated bone metastases.

In the clinical setting, the benefit of dovitinib treatment has been investigated for various cancer types including renal cell carcinoma [17,18], melanoma [19], endometrial cancer [20], breast cancer [21,22], gastrointestinal stromal tumor [23], mesothelioma [24], and transitional cell carcinoma [25]. In most studies, dovitinib showed minimal to modest activity. A trial comparing dovitinib and sorafenib demonstrated similar efficacy in heavily treated renal cell carcinoma patients [18]. An involving endometrial cancer patients investigated the efficacy of dovitinib according to FGFR2 mutation. The efficacy of dovitinib was independent of mutation [20]. A recent phase II randomized study reported that dovitinib in combination with fulvestrant showed a trend to improve median PFS compared with fulvestrant alone in FGF pathway amplified subgroup (10.9 months vs. 5.5 months) [22]. Biomarker analyses in two revealed a decreased plasma VEGFR level from baseline after dovitinib treatment, while plasma FGF23, vascular endothelial growth 
factor, placental growth factor, or hepatocyte growth factor levels were elevated due to compensatory upregulation because of FGFR or VEGFR inhibition [18,19].

Somatic mutation of FGFR1 has not been revealed in human prostate cancer. Previous studies reported overexpression or amplification of FGFR, which may become a predictive biomarker, in human prostate cancer, especially CRPC $[7-9,16]$. We did not perform immunohistochemical staining or gene sequencing analysis of cancer tissue. Instead, we examined the level of FGFR2, VEGFR2, and FGF23 in serum. No predictive biomarker for response to dovitinib was identified. Interestingly, miR-15a and miR-16 are thought to suppress FGF-2 and FGFR1 axis [26]. Future studies should assess if miR-15a or miR-16 expression is progressively lost in blood or tissue with development and progression of CRPC. Tissue FGFR overexpression or amplification and serum or tissue miR-15a or miR-16 suppression could be candidate predictive markers of dovitinib and should be investigated.

During the course of this clinical trial, treatment options for CRPC patients became more diverse. The second-generation anti-androgens, enzalutamide and abiraterone, were demonstrated to significantly improve survival in men with metastatic CRPC before and after docetaxel treatment [12,2729]. Since these drugs have little toxicity and excellent efficacy, they have been quickly introduced into clinical practice. Future research for CRPC patients should involve these drugs.

There are some inevitable limitations to the study. The study population was heterogenous and included CRPC patients enrolled before docetaxel treatment $(20 \%)$ and after chemotherapy. Thus, it is very difficult to define the best appropriate role of dovitinib in CRPC. In addition, the number of patients who underwent blood sampling was too small to pick out meaningful markers.

To overcome these shortcomings, future investigations should strive to enroll a homogenous study population and could include a combination with current standard treatment, especially second-generation anti-androgens. Comprehensive genetic testing, especially next generation sequencing, including FGFR amplification should be used to clarify dovitinib response and resistance mechanisms.
In conclusion, dovitinib displays modest antitumor activity with manageable toxicities in men with metastatic CRPC. Especially, patients who are chemo-naïve benefit from dovitinib.

\section{Conflicts of Interest}

We thank Novartis for their kind donation of dovitinib for this study.

\section{Acknowledgments}

We thank the patients and their families who took part in this study, the coordinators, the investigators and Korean Cancer Study Group (KCSG).

This study was supported by a grant from the National R\&D Program for Cancer Control, Ministry of Health and Welfare, Republic of Korea (1720150).

\section{Author Details}

${ }^{1}$ Division of Oncology / Hematology, Department of Internal Medicine, Korea University Anam Hospital, Seoul, ${ }^{2}$ Division of Hematology-Oncology, Department of Medicine, Samsung Medical Center, Sungkyunkwan University School of Medicine, Seoul, ${ }^{3}$ Division of Hemato-Oncology, Department of Internal Medicine, Veterans Health Service Medical Center, Seoul, ${ }^{4}$ Division of Hematology-Oncology, Department of Internal Medicine, Soonchunhyang University Hospital, Soonchunhyang University College of Medicine, Seoul, ${ }^{5}$ Department of Internal Medicine, Chungnam National University School of Medicine, Daejeon, ${ }^{6}$ Division of Hematology/ Oncology, Department of Internal Medicine, Keimyung University Dongsan Medical Center, Daegu, ${ }^{7}$ Department of Internal Medicine, Seoul National University Hospital, Seoul, ${ }^{8}$ Department of Internal Medicine, Korea Cancer Center Hospital, Seoul, ${ }^{9}$ Dongnam Institute of Radiological and Medical Sciences, Busan, ${ }^{10}$ Department of Internal Medicine, Gyeongsang Institute of Health Sciences, Gyeongsang National University Changwon Hospital, Gyeongsang National University College of Medicine, Changwon, ${ }^{11}$ Department of Hemato-oncology, Yeungnam Medical Center, Daegu, ${ }^{12}$ Department of Oncology, Asan Medical Center, University of Ulsan College of Medicine, Seoul, Korea

\section{References}

1. Jemal A, Bray F, Center MM, Ferlay J, Ward E, Forman D. Global cancer statistics. CA Cancer J Clin. 2011;61:69-90.

2. Jung KW, Won YJ, Oh CM, Kong HJ, Lee DH, Lee KH, et al.
Cancer statistics in Korea: incidence, mortality, survival, and prevalence in 2014. Cancer Res Treat. 2017;49:292-305.

3. Gnanapragasam VJ, Robinson MC, Marsh C, Robson CN, 
Hamdy FC, Leung HY. FGF8 isoform b expression in human prostate cancer. Br J Cancer. 2003;88:1432-8.

4. Heer R, Douglas D, Mathers ME, Robson CN, Leung HY. Fibroblast growth factor 17 is over-expressed in human prostate cancer. J Pathol. 2004;204:578-86.

5. Giri D, Ropiquet F, Ittmann M. Alterations in expression of basic fibroblast growth factor (FGF) 2 and its receptor FGFR1 in human prostate cancer. Clin Cancer Res. 1999;5:1063-71.

6. Sahadevan K, Darby S, Leung HY, Mathers ME, Robson CN, Gnanapragasam VJ. Selective over-expression of fibroblast growth factor receptors 1 and 4 in clinical prostate cancer. J Pathol. 2007;213:82-90.

7. Acevedo VD, Gangula RD, Freeman KW, Li R, Zhang Y, Wang F, et al. Inducible FGFR-1 activation leads to irreversible prostate adenocarcinoma and an epithelial-to-mesenchymal transition. Cancer Cell. 2007;12:559-71.

8. Memarzadeh S, Xin L, Mulholland DJ, Mansukhani A, Wu H, Teitell MA, et al. Enhanced paracrine FGF10 expression promotes formation of multifocal prostate adenocarcinoma and an increase in epithelial androgen receptor. Cancer Cell. 2007;12:572-85.

9. Johansson A, Rudolfsson S, Hammarsten P, Halin S, Pietras K, Jones J, et al. Mast cells are novel independent prognostic markers in prostate cancer and represent a target for therapy. Am J Pathol. 2010;177:1031-41.

10. Sarker D, Molife R, Evans TR, Hardie M, Marriott C, Butzberger-Zimmerli $\mathrm{P}$, et al. A phase I pharmacokinetic and pharmacodynamic study of TKI258, an oral, multitargeted receptor tyrosine kinase inhibitor in patients with advanced solid tumors. Clin Cancer Res. 2008;14:2075-81.

11. Tannock IF, de Wit R, Berry WR, Horti J, Pluzanska A, Chi KN, et al. Docetaxel plus prednisone or mitoxantrone plus prednisone for advanced prostate cancer. N Engl J Med. 2004;351: 1502-12.

12. de Bono JS, Logothetis CJ, Molina A, Fizazi K, North S, Chu $\mathrm{L}$, et al. Abiraterone and increased survival in metastatic prostate cancer. N Engl J Med. 2011;364:1995-2005.

13. Plosker GL. Sipuleucel-T: in metastatic castration-resistant prostate cancer. Drugs. 2011;71:101-8.

14. Aragon-Ching JB, Jain L, Gulley JL, Arlen PM, Wright JJ, Steinberg SM, et al. Final analysis of a phase II trial using sorafenib for metastatic castration-resistant prostate cancer. BJU Int. 2009;103:1636-40.

15. Lamont FR, Tomlinson DC, Cooper PA, Shnyder SD, Chester JD, Knowles MA. Small molecule FGF receptor inhibitors block FGFR-dependent urothelial carcinoma growth in vitro and in vivo. Br J Cancer. 2011;104:75-82.

16. Armstrong K, Ahmad I, Kalna G, Tan SS, Edwards J, Robson $\mathrm{CN}$, et al. Upregulated FGFR1 expression is associated with the transition of hormone-naive to castrate-resistant prostate cancer. Br J Cancer. 2011;105:1362-9.

17. Angevin E, Lopez-Martin JA, Lin CC, Gschwend JE, Harzstark A, Castellano D, et al. Phase I study of dovitinib (TKI258), an oral FGFR, VEGFR, and PDGFR inhibitor, in advanced or metastatic renal cell carcinoma. Clin Cancer Res. 2013;19: 1257-68.

18. Motzer RJ, Porta C, Vogelzang NJ, Sternberg CN, Szczylik C, Zolnierek J, et al. Dovitinib versus sorafenib for third-line targeted treatment of patients with metastatic renal cell carcinoma: an open-label, randomised phase 3 trial. Lancet Oncol. 2014;15:286-96.

19. Kim KB, Chesney J, Robinson D, Gardner H, Shi MM, Kirkwood JM. Phase I/II and pharmacodynamic study of dovitinib (TKI258), an inhibitor of fibroblast growth factor receptors and VEGF receptors, in patients with advanced melanoma. Clin Cancer Res. 2011;17:7451-61.

20. Konecny GE, Finkler N, Garcia AA, Lorusso D, Lee PS, Rocconi RP, et al. Second-line dovitinib (TKI258) in patients with FGFR2-mutated or FGFR2-non-mutated advanced or metastatic endometrial cancer: a non-randomised, open-label, twogroup, two-stage, phase 2 study. Lancet Oncol. 2015;16:686-94.

21. Andre F, Bachelot T, Campone M, Dalenc F, Perez-Garcia JM, Hurvitz SA, et al. Targeting FGFR with dovitinib (TKI258): preclinical and clinical data in breast cancer. Clin Cancer Res. 2013;19:3693-702.

22. Musolino A, Campone M, Neven P, Denduluri N, Barrios CH, Cortes J, et al. Phase II, randomized, placebo-controlled study of dovitinib in combination with fulvestrant in postmenopausal patients with $\mathrm{HR}(+)$, HER2(-) breast cancer that had progressed during or after prior endocrine therapy. Breast Cancer Res. 2017;19:18.

23. Kang YK, Yoo C, Ryoo BY, Lee JJ, Tan E, Park I, et al. Phase II study of dovitinib in patients with metastatic and/or unresectable gastrointestinal stromal tumours after failure of imatinib and sunitinib. Br J Cancer. 2013;109:2309-15.

24. Laurie SA, Hao D, Leighl NB, Goffin J, Khomani A, Gupta A, et al. A phase II trial of dovitinib in previously-treated advanced pleural mesothelioma: The Ontario Clinical Oncology Group. Lung Cancer. 2017;104:65-9.

25. Milowsky MI, Dittrich C, Duran I, Jagdev S, Millard FE, Sweeney CJ, et al. Phase 2 trial of dovitinib in patients with progressive FGFR3-mutated or FGFR3 wild-type advanced urothelial carcinoma. Eur J Cancer. 2014;50:3145-52.

26. Musumeci M, Coppola V, Addario A, Patrizii M, MaugeriSacca M, Memeo L, et al. Control of tumor and microenvironment cross-talk by miR-15a and miR-16 in prostate cancer. Oncogene. 2011;30:4231-42.

27. Beer TM, Armstrong AJ, Rathkopf DE, Loriot Y, Sternberg CN, Higano CS, et al. Enzalutamide in metastatic prostate cancer before chemotherapy. N Engl J Med. 2014;371:424-33.

28. Scher HI, Fizazi K, Saad F, Taplin ME, Sternberg CN, Miller $\mathrm{K}$, et al. Increased survival with enzalutamide in prostate cancer after chemotherapy. N Engl J Med. 2012;367:1187-97.

29. Ryan CJ, Smith MR, de Bono JS, Molina A, Logothetis CJ, de Souza $\mathrm{P}$, et al. Abiraterone in metastatic prostate cancer without previous chemotherapy. N Engl J Med. 2013;368:138-48. 